\title{
Ultrasound-based quantification of submandibular space compliance as predictor for difficult intubation. A prospective observational preliminary study.
}

\section{Background and Goal of Study}

Ultrasonography might be a tool to assess airway difficulty ${ }^{1}$. However, no validated measurement of submandibular space compliance as part of airway assessment has been reported ${ }^{2}$.

We investigated whether two indices, derived from the ultrasound-based quantification of the distances between the hyoid bone and mandible (HMD) in neutral, sniffing, and maximal hyperextended positions, may be used to predict the occurrence of Cormack 3 and 4 grades during direct laryngoscopy.

\section{Materials and Methods}

After the Ethics Committee approval and signing the informed consent forms, fifty adult patients scheduled to undergo general anaesthesia with oro-tracheal intubation were prospectively included. Exclusion criteria were morbid obesity and rapid sequence inductions.

We measured the HMDs in neutral, sniffing, and maximal hyperextended positions, in mid-sagital plane, using a curvilinear ultrasound transducer.

We calculated the ratio between HMDs in sniffing and neutral postions ( Compl $_{1}$ ) and the ratio between the HMDs in maximal hyperextended and neutral positions $\left(\mathbf{C o m p l}_{2}\right)$. All intubations were performed in sniffing position, with Macintosh curved laryngoscope blades, with no external laryngeal manipulation. We used ROC (receiver operating characteristics) curve analysis.
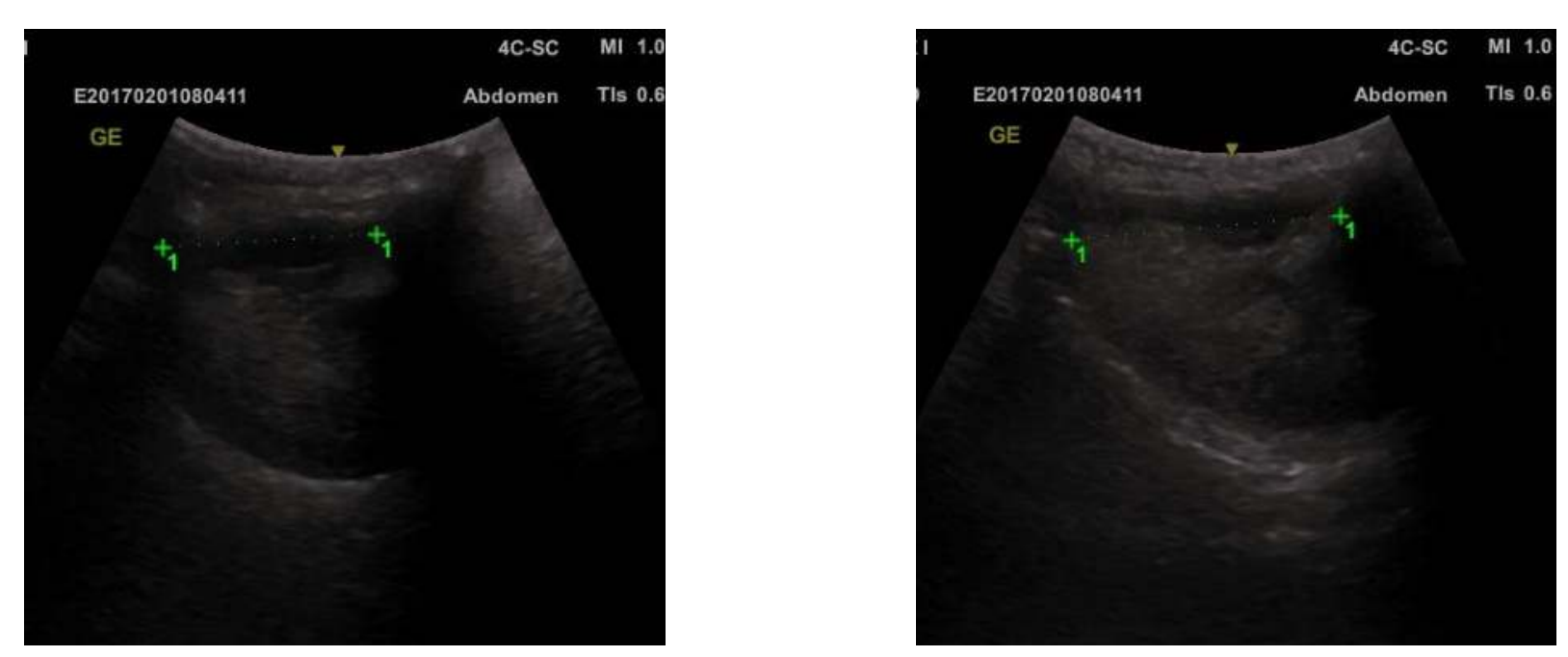

Compl $1=\frac{\text { HMD sniffing position }}{\text { HMD mental position }}$

Compl $2=\frac{\text { HMD maximal hyperextension }}{\text { HMD mental position }}$

$\mathrm{HMD}=$ hyo - mental distance

\section{Results and Discussion}

For Compl ${ }_{1}$, we identified an optimal threshold of 1.06, with $75 \%$ sensitivity, and 97.83 specificity. For Compl $_{2}$, we identified a threshold of 1.24 as being most disciminative between patients with and without difficult intubation, with $100 \%$ sensitivity and $71.7 \%$ specificity.

Thus, the lengthening of the submandibular space (HMD) by less than $24 \%$ from neutral to maximal hyperextended position had the highest sensitivity in predicting the occurrence of Cormack grades 3 and 4 . The areas under the ROC curve were 0.87 for $\mathrm{Compl}_{1}$ and 0.91 for $\mathrm{Compl}_{2}(\mathrm{p}=0.65)$ (Figure).

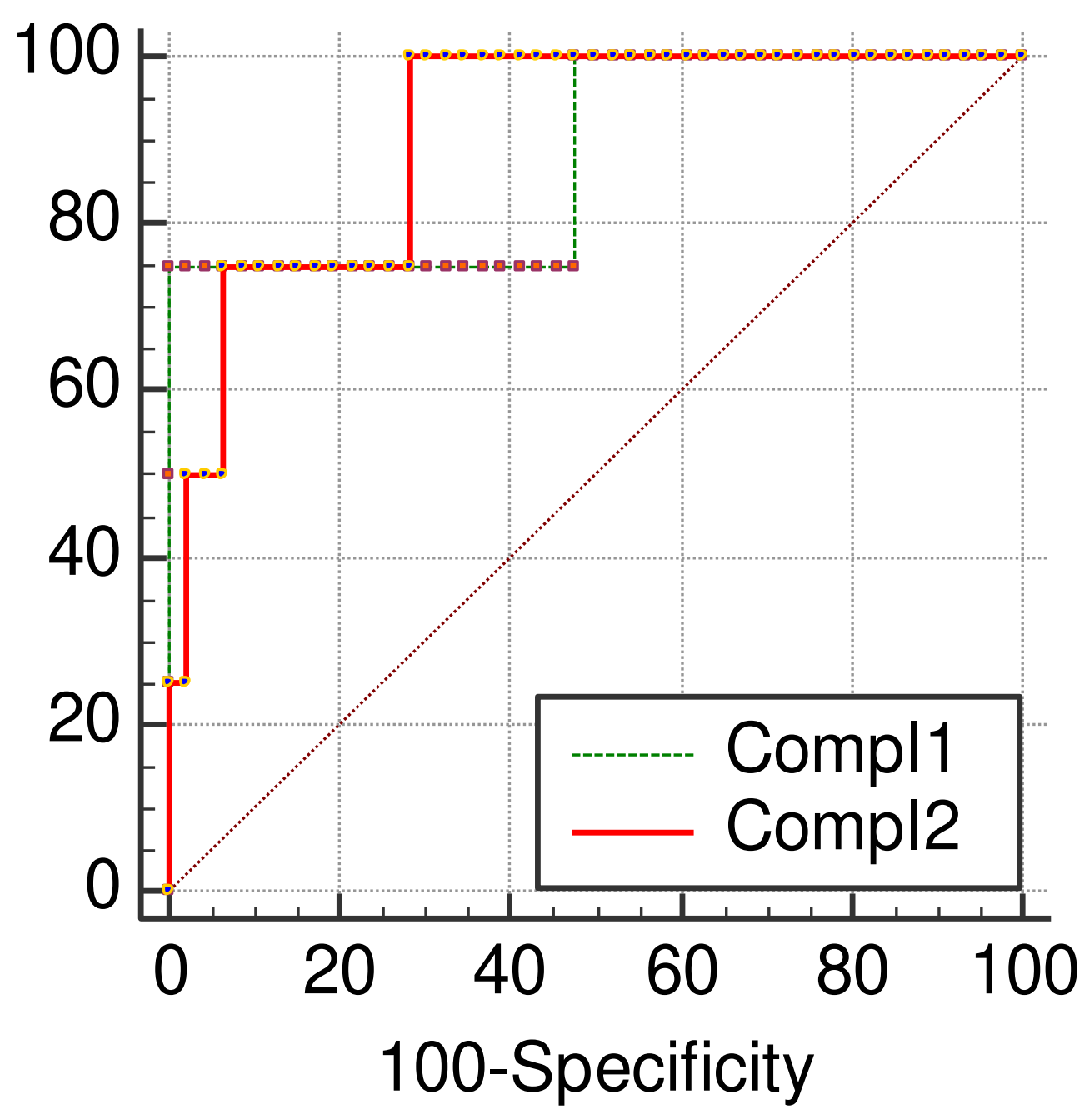

\section{Conclusion}

Ultrasound-based quantification of submandibular space compliance might be a useful tool, with good sensitivity and specificity, in the prediction of difficult airway.

\section{References}

${ }^{1}$ Fulkerson J, Moore H, Anderson T, Lowe R Jr. Ultrasonography in the preoperative difficult airway assessment. J Clin Monit Comput 2016; DOI 10.007/s10877-016-9888-7.

${ }^{2}$ Greenland KB. Airway assessment based on a three column model of direct laryngoscopy. Anaesth Intensive Care 2010;38:14-19. 